\title{
Ultrassonografia no auxílio do diagnóstico de vesiculite seminal em touros de corte ${ }^{1}$
}

\author{
Rui P.L. Oliveira ${ }^{2}$, Patricia C. Favaro ${ }^{2}$, Gabriel R. Pereira², Flávio A. Barca Jr², \\ Marcelo H.F. Borges ${ }^{2}$, Victor Hugo G. Galdioli ${ }^{2}$ e Celso Koetz Jr ${ }^{2 *}$
}

\begin{abstract}
Oliveira R.P.L., Favaro P.C., Pereira G.R., Barca Jr F.A., Borges M.H.F., Galdioli V.H.G. \& Koetz Jr C. 2018. [Ultrasonography in the diagnosis of seminal vesiculites in beef bulls.] Ultrassonografia no auxilio diagnóstico de vesiculite seminal em touros de corte. Pesquisa Veterinária Brasileira 38(6):1038-1042. Programa de Pós-Graduação em Saúde e Produção de Ruminantes, Laboratório de Produção Animal, Curso de Medicina Veterinária, Universidade Norte do Paraná, PR-218 Km 1, Jardim Universitário, Arapongas, PR 86702-670, Brazil. E-mail: celsokoetzjr@yahoo.com.br

The vesiculite is an inflammatory process of the vesicular glands, unilateral or bilateral, that affects bulls. The purpose of this study was to evaluate the use of ultrasound as a complementary method for early detection of changes in seminal vesicles in bulls. This study was conducted in the municipality of Videira, Santa Catarina. We evaluated 42 bulls, with an average age of 15 months, Aberdeen Angus and Polled Hereford breeds and in semi-confinement. Clinical animal examination and andrological exam were performed in all animals. Semen samples were obtained by electroejaculation and physical characteristics of the ejaculate were performed. Rectal palpation was performed by ultrasonography to evaluate vesicular glands changes. Statistical analysis of the data was performed using analysis of variance (ANOVA) for comparison between means with significance level of 5\%. The presence of vesiculitis was observed in 31 (73.8\%) from the 42 analyzed bulls. Of the 31 animals, 11 animals (35.5\%) presented bilateral vesiculitis and 20 showed unilateral vesiculitis $(64.5 \% ; \mathrm{P}<0.05)$. Animals with a larger scrotal perimeter tend to develop unilateral vesiculitis, which can be explained by the sexual precocity observed by sodomy among animals. In this way, the use of ultrasound helps preventively to detect animals with vesiculitis in breeding animals.
\end{abstract}

INDEX TERMS: Ultrasonography, seminal vesiculite, bulls, sexual precocity, sodomy, breeding soundness examination, clinics.

RESUMO.- A vesiculite é um processo inflamatório das glândulas vesiculares, podendo ser unilateral ou bilateral, que acomete reprodutores. $\mathrm{O}$ objetivo do presente trabalho foi avaliar a utilização da ultrassonografia como meio de diagnóstico precoce das alterações das vesículas seminais em touros. 0 presente trabalho foi realizado no município de Videira, Santa Catarina. Analisou-se um total 42 reprodutores, com média de idade de 15 meses, das raças Aberdeen Angus e Polled Hereford em semi-confinamento. Foi realizado exame clinico do estado geral dos animais e exame andrológico dos reprodutores. As amostras

\footnotetext{
${ }^{1}$ Recebido em 18 de maio de 2017.

Aceito para publicação em 30 de maio de 2017.

Dissertação de Mestrado do primeiro autor.

${ }^{2}$ Programa de Pós-Graduação em Saúde e Produção de Ruminantes, Laboratório de Produção Animal, Curso de Medicina Veterinária, Universidade Norte do Paraná (Unopar), PR-218 Km 01, Jardim Universitário, Arapongas, PR 86702-670, Brasil.*Autor para correspondência: celsokoetzjr@gmail.com
}

de sêmen para a realização do exame das características físicas do ejaculado foram obtidas por eletroejaculação. Através da palpação retal, realizou-se a avaliação das glândulas vesiculares por ultrassonografia. A análise estatística dos dados foi realizada por meio de análise de variância (ANOVA) para comparação entre médias com nível de significância de $5 \%$. A presença de vesiculite foi observada em 31 animais $(73,8 \%)$ dos 42 analisados. Dos 31 animais portadores 11 animais (35,5\%) apresentaram vesiculite bilateral e 20 apresentaram vesiculite unilateral $(64,5 \%$; $\mathrm{P}<0,05)$. Animais com perímetro escrotal maior tendem a desenvolver vesiculite unilateral, tal fato pode ser explicado pela precocidade sexual agravado pela sodomia entre os animais. O uso do ultrassom auxilia de forma preventiva a detecção de animais portadores de vesiculite em reprodutores.

TERMOS DE INDEXAÇÃO: Ultrassonografia, vesiculite seminal, touros, vesícula seminal, precocidade sexual, sodomia, exame andrológico, clínica. 


\section{INTRODUÇÃO}

Os fatores intrínsecos ao touro envolvem a avaliação da capacidade reprodutiva, a qual deve ser realizada de forma simplificada, mas que detecte o potencial do touro para a monta a campo para predizer de forma segura a fertilidade do rebanho com finalidade comercial (Parkinson 2004). Diante disso o reprodutor assume um papel importante no sistema de cria por representar um capital de investimento relevante (Menegassi et al. 2011, Evangelista 2015, Menegassi 2015). Touros jovens com maior perímetro escrotal apresentaram menor idade à puberdade e conhecer os padrões seminais da puberdade à maturidade sexual para animais que apresentam esse perfil genético pode subsidiar a seleção de animais geneticamente mais precoces (Lima 2009).

A incidência de vesiculite na população em geral de touros é de $1 \%$ a $10 \%$, no entanto a incidência de $20 \%$ até $49 \%$ já foram relatadas em animais confinados e mais jovens, quando o andrológico é realizado pela primeira vez (Hull \& Vogel 2008). Na maioria dos casos não há sinais externos da doença, porém observa-se que touros com vesiculite crônica apresentam arqueamento do posterior, dor ao evacuar ou a palpação transretal, e hesitação durante a monta. 0 diagnostico de vesiculite pode ser confirmado por exame de palpação transretal onde pode se perceber a vesícula alargada, irregular, assimétrica e frequentemente com a presença de tecido fibrótico (Givens \& Marley 2008). A presença de material purulento no ejaculado é um sinal clínico de vesiculite, porém não é patognomônico, outras patologias como epididimite, orquite ou postite podem contaminar com exsudato o ejaculado (Argue et al. 2013).

Dentre as várias afecções que podem cometer os reprodutores a vesiculite muitas vezes é negligenciada. A vesiculite é uma inflamação da vesicular seminal, que acomete bovinos maiores de 10 meses podendo ser de forma aguda ou crônica. Considerada uma significativa causa de perda econômica para o produtor, a vesiculite frequentemente resulta em descarte prematuro dos touros afetados e não tratados (Rovay et al. 2008), sendo a bacteria Actinomyces pyogenes o agente de maior frequência obtido a partir de infecções de glândulas vesiculares (Cavalieri \& Van Camp 1997). Portanto, o diagnóstico precoce é de suma importância para a saúde reprodutiva do animal, haja visto que isto pode interferir diretamente na qualidade espermática, diminuindo a motilidade sem afetar a morfologia espermática (Gnemmi \& Lefebvre 2009).

A avaliação reprodutiva dos machos com o uso do ultrassom não é realizada de forma rotineira, sendo um fator limitante para sua utilização o alto valor do equipamento. É também um fator limitante do seu uso a deficiência do ensino da técnica e da interpretação dos resultados nas escolas veterinárias, o que impede a sua utilização de forma clinica por profissionais do ramo. Entretanto, a ultrassonografia tem sido comercialmente utilizada para avaliação da composição de carcaça em animais vivos e, principalmente, para avaliação reprodutiva de fêmeas domésticas (Pinto et al. 2013, Hansar et al. 2014). 0 exame dos órgãos internos como as vesículas seminais, ampolas dos ductos deferentes e próstata são estruturas que podem ser identificadas e avaliadas pela técnica de ultrassonografia (Koetz Junior 2015).

O objetivo geral deste estudo foi verificar o uso do ultrassom como ferramenta no diagnóstico de alterações da vesícula seminal de touros das raças Angus e Polled Hereford, criados em sistema de semi-confinamento. Além disto, verificar as possíveis alterações dos parâmetros do exame andrológico nos animais portadores de vesiculite.

\section{MATERIAL E MÉTODOS}

Este estudo foi realizado de acordo com as normas de bem estar animal após aprovação pelo Comitê de Ética em Experimentação Animal (CEEA) da UNOPAR (Protocolo no. P009/16).

Local e animais. 0 trabalho foi realizado em dezembro de 2015 no município de Videira, Santa Catarina, com latitude $27^{\circ} 00^{\prime} 30^{\prime \prime} \mathrm{S}$ e longitude $51^{\circ} 09^{\prime} 06^{\prime \prime} \mathrm{W}$. Foi utilizado um grupo de 42 reprodutores, com idade de 12 meses até 18 meses, das raças Aberdeen Angus e Polled Hereford criados em sistema de semi-confinamento.

Coleta de amostras experimentais. No exame andrológico, a mensuração do perímetro escrotal foi realizada tracionando-se os testículos posteriormente a medindo-os com o auxílio de uma fita métrica, a qual deve circundar o maior diâmetro escrotal. Para obtenção dos ejaculados os touros foram contidos individualmente em tronco apropriado e utilizou-se o método de eletroejaculação (PulsatorIV - Lane USA). Após a coleta, foi realizado o exame imediato das características físicas do ejaculado onde se avaliou o movimento em massa dos espermatozoides (turbilhonamento, escala 1-5), a motilidade espermática progressiva retilínea (0-100\%) e o vigor espermático (escala 1-5). Além disto, uma amostra de sêmen foi acondicionada e estocada em formol salina tamponada a $1 \%$ para avaliação da morfologia espermática. Foram contabilizadas 200 células por ejaculado e mensurados em porcentagem os defeitos espermáticos conforme padrões preconizados pelo Colégio Brasileiro de Reprodução Animal (CBRA 2013).

Diagnóstico por ultrassonografia retal. Para a verificação ultrassonográfica das vesículas seminais foi utilizado aparelho de ultrassonografia transretal (Sonoscape A6 Vet) equipado com transdutor linear de $7,5 \mathrm{MHz}$. Os touros foram contidos e as fezes do reto foram removidas por meio de palpação transretal, após foi introduzido o transdutor lubrificado no reto e realizado a localização das glândulas acessória, com movimentos sobre a superfície dorsal das glândulas, foi possível a visualização transversal do eixo longitudinal da glândula.

Análise estatística. Os dados foram tabulados e analisados em duas etapas, primeiramente pela apresentação dos dados através da estatística descritiva e seguido pela comparação dos grupos através de análise de variância (ANOVA). As análises estatísticas foram realizadas utilizando o pacote estatístico Minitab versão 16 (State College, PA, USA: Minitab, Inc. 2010), sendo adotado nível mínimo de significância de 5\%.

\section{RESULTADOS E DISCUSSÃO}

A quantidade de touros com vesiculite foi de 31 animais $(73,8 \%)$ dos 42 analisados. Dos 31 animais portadores 11 animais $(35,5 \%)$ apresentaram vesiculite bilateral, outros 20 apresentaram vesiculite unilateral $(64,5 \%)$. Hull \& Vogel (2008) demonstraram que a incidência de vesiculite em animais mais jovens e maiores de 9 anos é mais frequente e que na população em geral está entre $1 \%$ a $10 \%$ e em que animais criados sob o sistema de confinamento, apresentam incidência entre 20\% a 49\%. Essa divergência pode ser explicada pelo fato de que no presente trabalho houve a utilização de ultrassonografia como ferramenta auxiliar ao diagnóstico o que possibilitou uma maior precisão diagnóstica. Portanto, a ultra-sonografia é uma das ferramentas diagnósticas com 
imagens tomográficas em tempo real muito utilizadas na medicina moderna para visualizar órgãos internos, seu tamanho, estrutura e lesões (Pechman \& Eilts 1987, Pierson et al. 1988).

As glândulas sem alterações apresentaram superfície regular, com lóbulos isoecóicos separados por regiões hipoecóicas e circundando todo perímetro da glândula uma membrana hiperecóica (Fig.1). As imagens ultrassonográficas encontradas são semelhantes as produzidas por Chacur et al. (2001), não evidenciando alterações graves na estrutura vesicular. Durante a realização do exame de palpação transretal,
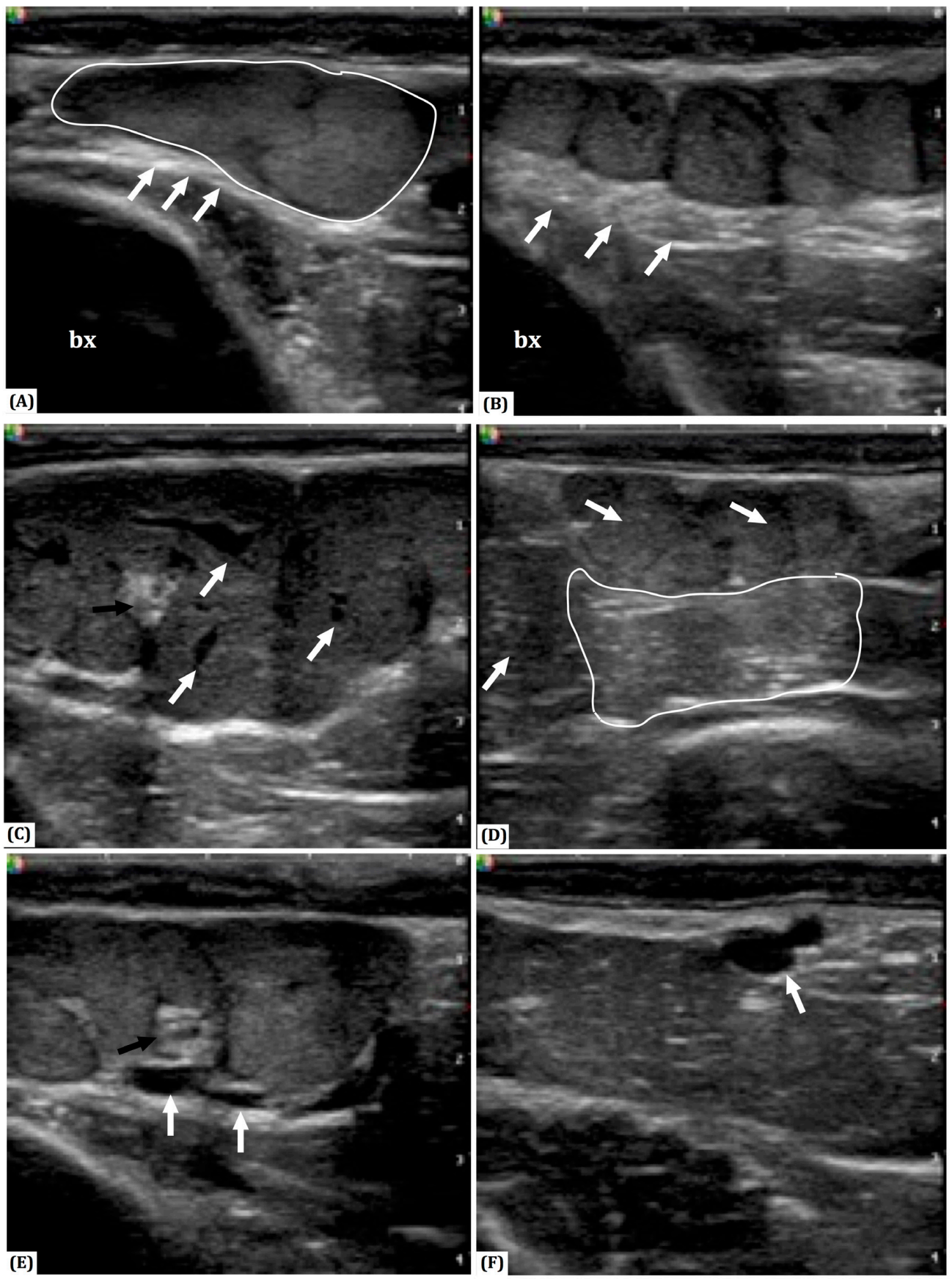

Fig.1. Imagens ultrassonográficas de vesículas seminais em touros. (A) Visualização ultrassonográfica da vesícula seminal (contorno branco) normal de touros que apresenta parede bem definida, hiperecóica e homogênea (setas branca), bexiga (bx). (B) Vesícula seminal com a presença característica de lóbulos glandulares, presença da parede hiperecóica homogênea e levemente espessa (setas branca), bexiga (bx). (C) Visualização ultrassonográfica de vesícula seminal com alterações, presença de lóbulos de tamanho alterado, abscesso (seta preta), fluido interlobular com a presença de areas anecóicas (setas brancas), membrana inferior da glândula vesicular apresenta parede hipoecóica e irregular. (D) Área de fibrose (contorno branco), lóbulos apresentam características de hipo a hiperecóica (setas brancas), membrana da glândula apresenta parede hipoecóica irregular. (E) Presença de fluído extralobular com áreas anecóicas (setas brancas) na região inferior da glândula, abscesso (seta preta). (F) Glândula sem a presença de glóbulos característicos e bem definidos, presença de areas hipoecóicas com aspecto desuniforme, presença de cisto na porção dorsal da vesícula (seta branca). 
Quadro 1. Análise dos parâmetros relativos ao exame do ejaculado, morfologia espermática e perímetro escrotal durante a avaliação andrológica em reprodutores com presença de vesiculite

\begin{tabular}{|c|c|c|c|c|c|c|c|c|}
\hline Vesiculite & $\begin{array}{l}\text { Animais } \\
\text { (n) }\end{array}$ & $\begin{array}{c}\text { Perímetro } \\
\text { Escrotal } \\
(\mathrm{cm}) \\
\end{array}$ & $\begin{array}{c}\text { Motilidade } \\
\text { (\%) }\end{array}$ & $\begin{array}{l}\text { Turbilhão } \\
\text { (1-5) }\end{array}$ & $\begin{array}{l}\text { Vigor } \\
(1-5)\end{array}$ & $\begin{array}{c}\text { Defeitos Maiores } \\
(\%)\end{array}$ & $\begin{array}{c}\text { Defeitos } \\
\text { Menores } \\
(\%)\end{array}$ & $\begin{array}{c}\text { Defeitos Totais } \\
\text { (\%) }\end{array}$ \\
\hline Ausente & 11 & $36,27 \pm 2,72^{\mathrm{ab}}$ & $77,27 \pm 18,89$ & $3,82 \pm 1,60$ & $4,00 \pm 0,77$ & $25,00 \pm 24,00$ & $9,00 \pm 8,04$ & $34,00 \pm 26,45$ \\
\hline Unilateral & 20 & $37,55 \pm 2,82^{\mathrm{a}}$ & $77,25 \pm 24,14$ & $3,75 \pm 1,45$ & $3,95 \pm 1,05$ & $17,00 \pm 11,41$ & $10,80 \pm 7,64$ & $27,80 \pm 17,10$ \\
\hline$p$-valor & - & 0,049 & 0,895 & 0,968 & 0,980 & 0,457 & 0,701 & 0,733 \\
\hline
\end{tabular}

Médias seguidas de letras diferentes na mesma coluna diferem estatisticamente entre si $(\mathrm{P}<0,05)$.

observou-se achados semelhantes aos de Nascimento \& Santos (2011), onde detectaram aparência das glândulas vesiculares aumentadas de volume, hiperêmica e consistente à palpação retal. Os equipamentos utilizados em exames de campo, via de regra, possuem modo de luminosidade do tipo B-mode e exibem as imagens em pontos bidimensionais, sendo que as estruturas anatômicas são apresentadas de forma transversal (Cartee et al.1989). Muito embora a ultrassonografia eco-doppler, que produz uma imagem bidimensional de alta resolução com mapeamento capilar a cores, ainda não seja utilizada em touros, ela permite uma melhor visualização principalmente das alterações de fluxo, resistência e pressão vascular (Cruz \& Freitas 2001). No entanto, embora o equipamento de ultrasom esteja amplamente disponível para examinar o trato reprodutivo em reprodutores, é realizado com menor frequência, tipicamente quando algum problema já em andamento ou exige um diagnóstico preciso de patologias das glândulas vesicais.

A medida dos perímetros escrotais encontrados no presente trabalho (Quadro 1) nos animais portadores de vesiculite unilateral foi de $37,55 \mathrm{~cm}$, sendo maior que a medida verificada por Knights et al. (1984), que foi de $35,7 \mathrm{~cm}$ em animais da mesma raça, idade e hígidos. As glândulas vesicais não desempenham função de nutrição e reservatório de espermatozóides e sim a de secretar o líquido seminal no qual estão contidas as células espermáticas. Portanto, o uso da ultrassonografia como ferramenta auxiliar do exame andrológico, vem se tornando um método importante como teste complementar, especialmente no caso desordens subclínicas (Gábor et al. 1998, Martínez \& Barth 2007), entretanto esta técnica permite o exame do aparelho reprodutivo dos touros sem risco a sua integridade reprodutiva (Koetz Junior 2015).

Dentre os parâmetros estudados, houve correlação entre animais portadores de vesiculite com o perímetro escrotal $(\mathrm{P}<0,05)$. Os animais com maior perímetro escrotal apresentavam maior incidência de vesiculite unilateral de acordo com o demonstrado por Lima (2009). 0 fato dos animais com maior perímetro escrotal apresentarem maior incidência de vesiculite unilateral, pode ser explicado, pelo fato destes animais serem mais precoces sexualmente. Ao atingirem a puberdade esses animais apresentam uma maior libido e assim maior atividade sexual o que pode leva-los praticar à sodomia nos contemporâneos submissos. Entretanto, não foram encontradas correlações significativas entre a atividade copulatória e o comprimento ou espessura vesicular em reprodutores da raça Santa Gertrudes (Rocha et al. 1994). o comportamento sexual anormal pode ocasionar lesões no pênis e consequente uma infecção ascendente, por meio da uretra, especialmente quando há alta concentração de machos no mesmo local (Lunstra et al. 1978, Gottschall \& Mattos 1997, Vasconcelos 2001).

Os animais portadores de vesiculites bilaterais apresentaram menor perímetro escrotal quando comparados aos sem alterações, isto pode ser explicado por uma possível infeção crônica das glândulas seminais. Os animais com vesiculite apresentaram glândulas enrijecidas, aumentadas na palpação transretal e áreas de hiperecogenicidade característicos de lesão ao exame ultrassonográfico (Fig.1). A presença de vesículas seminais maiores foi associada com o tamanho da circunferência escrotal e maior anormalidades de morfologia espermática, em especial gota proximal e anormalidades da peça central em touros jovens (Berry et al. 1983). As médias dos parâmetros seminais obtidos pelo exame andrológico estão dentro do esperado para idade, pois estes valores são próximos aos estabelecidos pelo CBRA. De acordo com Cavalieri \& Van Camp (1997) a única alteração que ocorrem em animais portadores de vesiculite é a diminuição da motilidade, o que também foi constado por Dargatz et al. (1987) em 80\% dos animais. Diferentemente dos resultados obtidos no presente trabalho onde a motilidade não apresentou variação entre indivíduos afetados e não afetados.

Portanto, o diagnóstico das patologias do sistema reprodutor masculino pode muitas vezes ser frustrante devido ao desafio envolvido na determinação precisa do local, gravidade e prognóstico. A introdução do exame ultra-sonográfico complementar permite que os clínicos abordem estas questões importantes em todos os touros de fazenda. Os resultados deste estudo sugerem que a avaliação de rotina do trato reprodutivo de reprodutores deve-se incluir o ultrassom da genitália interna para uma avaliação detalhada do potencial reprodutivo desses animais.

\section{CONCLUSÕES}

A incidência de vesiculite unilateral foi maior em touros jovens com maior perímetro escrotal das raças Aberdeen Angus e Polled Hereford.

A utilização da técnica de ultrassonografia se mostrou uma ferramenta útil no diagnóstico das alterações das vesículas seminais em touros.

Agradecimentos.- Pelo apoio financeiro à Diretoria de Pesquisa da UNOPAR/Kroton Educacional, à Coordenação de Aperfeiçoamento de Pessoal de Nível Superior (CAPES/PNPD), ao Conselho Nacional de Desenvolvimento Científico e Tecnológico (CNPq/Universal no. 456724/2014-1), e ao Três Marias Agronegócio. 


\section{REFERÊNCIAS}

Argue B., Chousalkar K.K. \& Chenoweth P.J. 2013. Presence of ureaplasma diversum in the Australian cattle population. Aust. Vet. J. 91(3):99-101. http://dx.doi.org/10.1111/avj.12009. PMid:23438461.

Berry J.G., Brinks J.S. \& Russell W.C. 1983. Relationship of seminal vesicle-size and measures of libido in yearling beef bulls. Theriogenology 19(2):279284. http://dx.doi.org/10.1016/0093-691X(83)90014-6. PMid:16725795.

Cartee R.E., Gray B.W., Powe T.A., Hudson R.S. \& Whitesides J. 1989. Preliminary implications of B-mode ultrasonography of the testicles of beef bulls with normal breeding soundness examinations. Theriogenology 31(6):11491157. http://dx.doi.org/10.1016/0093-691X(89)90083-6. PMid:16726632.

Cavalieri J. \& Van Camp S.D. 1997. Bovine seminal vesiculitis: a review and update. Vet. Clin. N. Am., Food Anim. Pract. 13(2):233-241. http://dx.doi. org/10.1016/S0749-0720(15)30337-6. PMid:9216045.

CBRA 2013. Manual para Exame Andrológico e Avaliação de Sêmen Animal. 3 a ed. Colégio Brasileiro de Reprodução Animal, Belo Horizonte. 104p.

Chacur M.G.M., Peres J.A., Luz M.R. \& Giuffrida R. 2001. Importance of ultrasonography in diagnosis of seminal vesiculitis and acrobustitis in bulls. Revta Bras. Reprod. Anim. 25(2):260-262.

Cruz J.F. \& Freitas V.J.F. 2001. A ultrassonografia em tempo real na reprodução de caprinos. Ciência Animal 11(1):53-61.

Dargatz D.A., Mortimer R.G. \& Ball L. 1987. Vesicular adenitis of bulls: a review. Theriogenology 28(4):513-521. http://dx.doi.org/10.1016/0093691X(87)90256-1. PMid:16726334.

Evangelista G.T. 2015. Análise da comercialização de touros de corte no Rio Grande do Sul. Dissertação de Mestrado, Programa de Pós-graduação em Zootecnia, Faculdade de Agronomia, Universidade Federal do Rio Grande do Sul, Porto Alegre, RS. 66p.

Gábor G., Sasser R.G., Kastelic J.P., Mézes M., Falkay G., Bozó S., Csík J.V., Bárany I., Hidas A., Szász Junior F. \& Boros G. 1998. Computer analysis of video and ultrasonographic images for evaluation of bull testes. Theriogenology 50(2):223-228. http://dx.doi.org/10.1016/S0093-691X(98)00129-0. PMid:10734489.

Givens M.D. \& Marley M.S.D. 2008. Pathogens that cause infertility of bulls or transmission via semen. Theriogenology 70(3):504-507. http://dx.doi. org/10.1016/j.theriogenology.2008.05.033. PMid:18501958.

Gnemmi G. \& Lefebvre R.C. 2009. Ultrasound imaging of the bull reproductive tract: an important field of expertise for veterinarians. Vet. Clin. N. Am. Food Anim. Pract. 25(3):767-779. http://dx.doi.org/10.1016/j.cvfa.2009.07.006. PMid:19825443.

Gottschall C.S. \& Mattos R.C. 1997. Achados de exames andrológicos em touros de corte Bos taurus e Bos indicus. Revta Bras. Reprod. Anim. 21:25-28.

Hansar E., Lemma A. \& Yilma T. 2014. Pre-service ultrasonic and manual evaluation of the reproductive organs of dairy cows presumed to be in estrus. SpringerPlus 3(1):529. http://dx.doi.org/10.1186/2193-18013-529. PMid:25279320.

Hull B.L. \& Vogel S.R. 2008. Seminal vesiculitis. Vet. Clin. N. Am., Food Anim. Pract. 24(2):267-272. http://dx.doi.org/10.1016/j.cvfa.2008.02.006. PMid:18471568.
Knights S.A., Baker R.L., Gianola D. \& Gibb J.B. 1984. Estimates of heritabilities and of genetic and phenotypic correlations among growth and reproductive traits in yearling Angus bulls. J. Anim. Sci. 58(4):887-893. http://dx.doi. org/10.2527/jas1984.584887x. PMid:6725151.

Koetz Junior C. 2015. Avaliação ultrassonográfica do potencial reprodutivo de touros, p.119-123. In: Ibid. (Ed), Aspectos Reprodutivos do Touro: Teoria e Prática. Agrolivros, Guaíba.

Lima F.P.C. 2009. Puberdade em tourinhos da raça Nelore avaliada pelo perímetro escrotal, características seminais e endócrinas. Tese de Doutorado, Escola de Veterinária Universidade Federal de Minas Gerais, Belo Horizonte. 65p.

Lunstra D.D., Ford J.J. \& Echternkamp S.E. 1978. Puberty in beef bulls: hormone concentration, growth, testicular development, sperm production and sexual aggressiveness in bulls of different breeds. J. Anim. Sci. 46(4):1054-1062. http://dx.doi.org/10.2527/jas1978.4641054x. PMid:566747.

Martínez M.F. \& Barth A.D. 2007. Early detection and treatment of vesicular adenitis in bulls. Anim. Reprod. Sci. 101(3/4):252-256. http://dx.doi. org/10.1016/j.anireprosci.2006.09.009. PMid:17027202.

Menegassi S.R.O. 2015. O impacto econômico da avaliação reprodutiva de touros, p.41-43. In: Ibid. (Ed), Aspectos Reprodutivos do Touro: teoria e prática. Agrolivros, Guaíba.

Menegassi S.R.O., Barcellos J.O.J., Peripolli V., Pereira P.R.R.X., Borges J.B.S. \& Lampert V.N. 2011. Measurement of scrotal circumference in beef bulls in Rio Grande do Sul. Arq. Bras. Med. Vet. Zootec. 63(1):87-93. http://dx.doi. org/10.1590/S0102-09352011000100014.

Nascimento E.F. \& Santos R.L. 2011. Patologias das glândulas sexuais acessórias, p.107-110. In: Ibid. (Eds), Patologia da Reprodução dos Animais Domésticos. $3^{\mathrm{a}}$ ed. Guanabara Koogan, Rio de Janeiro.

Parkinson T.J. 2004. Evaluation of fertility and infertility in natural service bulls. Vet. J. 168(3):215-229. http://dx.doi.org/10.1016/j.tvjl.2003.10.017. PMid:15501139.

Pechman R.D. \& Eilts B.E. 1987. B-mode ultrasonography of the bull testicle. Theriogenology 27(2):431-441. http://dx.doi.org/10.1016/0093691X(87)90231-7. PMid:16726248.

Pierson R.A., Kastelic J.P. \& Ginther O.J. 1988. Basic principles and techniques for transretal ultrasonography in cattle and horses. Theriogenology 29(1):320. http://dx.doi.org/10.1016/0093-691X(88)90028-3.

Pinto L.F.B., Tarouco J.U., Pedrosa V.B., Jucá A.F., Leão A.G. \& Moita A.K.F. 2013. Live weight, carcass ultrasound images, and visual scores in Angus cattle under feeding regimes in Brazil. Trop. Anim. Health Prod. 45(6):12811287. http://dx.doi.org/10.1007/s11250-013-0358-7. PMid:23341195.

Rocha A., Carpenter B.B., Hawkins H.E., Sprott L.R. \& Forrest D.W. 1994. Relationship between seminal vesicle size and copulatory activity in bulls. Theriogenology 42(7):1177-1181. http://dx.doi.org/10.1016/0093691X(94)90866-4. PMid:16727622.

Rovay H., Barth A.D., Chirino-Trejo M. \& Martinez M.F. 2008. Update on treatment of vesiculitis in bulls. Theriogenology 70(3):495-503. http:// dx.doi.org/10.1016/j.theriogenology.2008.05.002. PMid:18514806.

Vasconcelos C.O.P. 2001. Estádio de maturidade sexual em touros da raça Nelore, dos 20 aos 22 meses de idade. Dissertação de Mestrado, Universidade Federal de Viçosa, Viçosa. 59p. 